\title{
Transarterial chemoembolization for the treatment of hepatocellular carcinoma: a review
}

This article was published in the following Dove Press journal:

Journal of Hepatocellular Carcinoma

27 July 2017

Number of times this article has been viewed

\author{
Rodolfo Sacco' \\ Gherardo Tapete' \\ Natalia Simonetti' \\ Rossella Sellitri' \\ Veronica Natali' \\ Sara Melissari' \\ Giuseppe Cabibbo² \\ Lilia Biscaglia ${ }^{3}$ \\ Giampaolo Bresci' \\ Luca Giacomelli ${ }^{4}$ \\ 'Department of Gastroenterology, \\ Pisa University Hospital, Pisa, \\ ${ }^{2}$ University of Palermo, Palermo, \\ ${ }^{3}$ Department of Public Health \\ and Infectious Diseases, Sapienza \\ University of Rome, Rome, \\ ${ }^{4}$ Department of Surgical Sciences and \\ Integrated Diagnostics, University of \\ Genoa, Genoa, Italy
}

Correspondence: Rodolfo Sacco

Department of Gastroenterology, Pisa

University Hospital, Via Paradisa 2, 56I24

Pisa, Italy

Tel $+390509974 I I$

Fax+39050997412

Email r.sacco@ao-pisa.toscana.it

\begin{abstract}
According to the current European Association for the Study of Liver guidelines, transarterial chemoembolization (TACE) is the recommended first-line therapy for patients with intermediate-stage (Barcelona Clinic Liver Cancer-B class) hepatocellular carcinoma (HCC). The efficacy of this therapy is supported by robust evidence; however, there is still a lack of standardization in treatment methodology, and TACE protocols are widely variable. Moreover, TACE can be associated with a number of contraindications. Despite these limitations, research on TACE is still ongoing with the aim of optimizing the use of this methodology in the current management of HCC. In particular, TACE represents a control in comparative studies, and it is currently being investigated in combination schemes, for example, with sorafenib. In this review, we briefly describe the current scenario and the clinical innovations regarding TACE for the treatment of HCC.

Keywords: TACE, HCC, BCLC-B, sorafenib
\end{abstract}

\section{Introduction}

According to the guidelines issued by the European Association for the Study of Liver, patients affected from hepatocellular carcinoma (HCC) can be classified and treated according to the Barcelona Clinic Liver Cancer (BCLC) staging system. ${ }^{1}$ In particular, the first-line therapy recommended for patients with intermediate-stage (BCLC-B class) HCC is transarterial chemoembolization (TACE). ${ }^{1}$ The efficacy of this procedure is supported by robust evidence. ${ }^{2,3}$ However, there is still a lack of standardization in treatment methodology, and TACE protocols are widely variable., ${ }^{4,5}$ Moreover, the long-term outcomes of patients managed with TACE remain poor, ${ }^{6}$ and TACE can be associated with a number of contraindications. ${ }^{4}$ Of note, patients with BCLC-B HCC present highly heterogeneous features, and therefore, the behavior of intermediatestage HCC patients is difficult to anticipate. ${ }^{7,8}$ Indeed, BCLC-B stage includes large $(>5 \mathrm{~cm})$ or multinodular HCC with no vascular invasion, in patients with good Eastern Cooperative Oncology Group (ECOG) Performance Status and Child-Pugh class A or B. This group of patients is rather heterogeneous because it includes tumors characterized by different extension (ie, large monofocal unresectable HCCs, multifocal small HCC, and extensive tumors with subtotal replacement of liver parenchyma), different stages of liver function (from compensated cirrhosis in A5 Child-Pugh class to decompensated liver disease in B9 Child-Pugh class), and presence or absence of portal vein thrombosis, but no macrovascular invasion. Therefore, the development of 
an effective treatment strategy for all patients with BCLC-B $\mathrm{HCC}$ does not seem to be an easy task: a tailored approach is required to optimize the clinical outcomes in each patient. ${ }^{9,10}$

In this complex scenario, research on TACE is still ongoing with the aim of optimizing the use of this methodology in the current management of HCC. In particular, TACE represents a control for comparative studies, and it is currently being investigated into combination schemes, for example, with sorafenib. ${ }^{9}$

In this review, we briefly describe the current scenario and the clinical innovations regarding TACE for the treatment of $\mathrm{HCC}$.

\section{Search strategy}

With the aim of identifying the key publications specifically addressing the efficacy and safety of the TACE in HCC patients, a literature search was conducted on the MEDLINE database using the search terms "hepatocellular carcinoma," "liver cancer," and "primary liver carcinoma," either individually or in combination with the terms "randomized," "controlled clinical trials," "clinical trials," "Phase III studies," "meta-analysis," "combined therapy," "treatment," "liver cancer," "TACE," "TAE," "chemoembolization," "embolization," and "locoregional treatments," as well as a manual search and review of reference lists were done. The search was limited to studies published in English, and only the studies published in 2015 and 2016 were considered. Studies were selected for inclusion according to their relevance for the topic, in line with the authors' judgment.

\section{TACE in head-to-head studies}

The different efficacy of conventional TACE (cTACE) and drug-eluting bead TACE (DEB-TACE; developed to enhance drug delivery to the tumor and reduce its systemic availability) remains a matter of debate. In a retrospective database study, Kloeckner et al compared the overall survival (OS) of HCC patients who were treated with cTACE $(n=174)$ and DEB-TACE $(n=76) .{ }^{11}$ These two groups showed similar baseline characteristics. Patients on DEB-TACE received a lower number of treatment sessions compared with those on cTACE ( 2.9 vs $4.0 ; p=0.01)$. The OS was comparable and equal to 13.6 months (95\% CI: $10.7-16.2)$ in the cTACE group and 12.3 months $(95 \%$ CI: 10.3-19.6) in the DEB-TACE group. This similar OS was also consistent when stratifying patients according to Child-Pugh stage. The authors of this study concluded that DEB-TACE can be considered a more appealing treatment option compared cTACE, because only a lower number of treatment sessions are required to achieve similar survival outcomes.

Liu et al have investigated the optimal therapeutic regimen for TACE by comparing the efficacy of chemoembolization with different anticancer agents in a single-blind, three-group randomized trial. ${ }^{12}$ Group 1 received singledrug (doxorubicin) chemoembolization $(n=50)$, whereas Group 2 received double-drug (doxorubicin and mitomycin C) chemoembolization $(n=59)$. Patients in Group 3 were treated with triple-drug (doxorubicin, mitomycin $\mathrm{C}$, and gemcitabine) chemoembolization $(n=53)$. The OS and time to progression (TTP) in Groups 1, 2, and 3 were 14.9 and 6.4, 13.2 and 6.4, and 20.5 and 6.8 months, respectively. Overall response rates (ORRs) for these three groups were $22.0 \%, 40.7 \%$, and $56.6 \%$, respectively. Therefore, TACE with multiple chemotherapeutic agents might significantly improve survival and tumor response; in addition, gemcitabine seems to be associated with a further improvement in prognosis.

Selective internal radiation therapy (SIRT) has gained mounting attention in the treatment of HCC. ${ }^{13}$ In the SIRTACE open-label, multicenter, randomized pilot study, the safety and health-related quality of life (HRQoL) changes followed by TACE and SIRT were evaluated. ${ }^{13}$ Furthermore, patients with unresectable $\mathrm{HCC}$, Child-Pugh $\leq \mathrm{B} 7, \mathrm{ECOG}$ performance status $\leq 2$, and $\leq 5$ liver lesions with no extrahepatic spread were randomly assigned to either TACE (at 6-week intervals; $n=15$ ) or single-session SIRT (yttrium-90 resin microspheres; $n=13$ ). Both these treatments were well tolerated. At Week 12, neither treatment had any effect on HRQoL as measured by Functional Assessment of Cancer Therapy-Hepatobiliary total or its subscales. The ORRs were $13.3 \%$ with TACE and $30.8 \%$ with SIRT; the disease control rates (DCRs) were $73.3 \%$ and $76.9 \%$, respectively. Two patients in each group were downstaged for liver transplantation or radiofrequency ablation.

In a similar randomized study, DEB-TACE was compared with SIRT. ${ }^{14}$ In total, 12 patients were assigned to each group. TACE was repeated every 6 weeks until no viable tumor tissue was detected by using magnetic resonance imaging or contraindications prohibited further treatment. SIRT could be repeated once in case of recurrence. Median progression-free survival (PFS) was comparable in the two groups (7.2 months with TACE [25\%-75\% percentile 2.9/11.8] and 6.0 months [4.0/13.8] with SIRT; $p=0.61)$. Similarly, median TTP and OS were comparable (TTP $=11.2$ months [3.0-20.3] vs 12.3 months [4.4-18.7], $p=0.57$; OS $=26.2$ [5.9-31.6] vs 
19.7 months [6.4-not reached], $p=0.92$ ). Seven patients died in each group. Causes of death were liver failure $(n=4$ in SIRT group), tumor progression ( $n=4$ in TACE group), cardiovascular events ( $\mathrm{n}=1$ in each group), and undetermined ( $n=1$ in each group).

The similar efficacy of TACE (especially cTACE) and SIRT was confirmed in a prospective observational study on 86 patients. ${ }^{15}$ Furthermore, patients with BCLC-B HCC received either TACE $(n=42)$ or SIRT. Median OS (TACE: 18 months; SIRT: 16.4 months) and median TTP (6.8 vs 13.3 months) were comparable. However, the number of treatment sessions, the average rate of treatment sessions per patient, total hospitalization time, and the rate of adverse events were higher with TACE.

Last, an interesting observational study, although with a retrospective design, from the large Italian Liver Cancer group database has evaluated 485 BCLC-B patients diagnosed with naïve HCC after 1999. ${ }^{16}$ Treatment distribution was as follows: TACE $(n=233,51.1 \%)$, curative treatments $(n=145,31.8 \%)$, sorafenib $(n=18,3.9 \%)$, other $(n=39,8.5 \%)$, and best supportive care (BSC; $n=21,4.6 \%)$. Twenty-nine patients were lost to follow-up. Median OS was 45 months (95\% CI: 37.4-52.7) for curative treatments, 30 months (95\% CI: 24.7-35.3) for TACE, 14 (95\% CI: 10.5-17.5) for sorafenib, 14 months (95\% CI: 5.2-22.7) for other treatments, and 10 months (95\% CI: $6.0-14.2)$ for BSC $(p<0.0001)$. Of note, curative treatments reduced mortality (hazard ratio [HR] $=0.197,95 \%$ CI: 0.098-0.395) more compared with TACE (HR $=0.408,95 \%$ CI: $0.211-0.789 ; p<0.0001)$ as compared with BSC. These findings were confirmed with propensity score matching.

\section{TACE within combination schemes}

The combination of TACE and sorafenib has been extensively investigated. 5,9 This approach is based on a strong mechanistic rationale. In fact, TACE determines hypoxia and necrosis of tumor tissue, increasing the concentration of angiogenic growth factors such as vascular endothelial growth factor and insulin-like growth factor 2 , which contribute to disease progression. ${ }^{17}$ Noteworthy, the degree of hypoxia induced by TACE varies according to the liver segment to which TACE is applied. ${ }^{9}$ The growth effects on the tumor induced by the angiogenic factors can be blocked by sorafenib, eventually improving clinical outcome. ${ }^{18}$

In an open-label trial published in 2015, Cosgrove et al have evaluated the efficacy of the combination of DEB-TACE and sorafenib in 54 naïve patients with a Child-Pugh score of A-B7 (62\% in BCLC-C stage). ${ }^{19}$ Sorafenib therapy was started 1 week before the first DEB-TACE procedure. Up to four rounds of DEB-TACE therapy were allowed on demand within 6 months. The 6-month DCR was 94\%; median TTP and OS were 13.9 and 20.4 months, respectively. The wide majority of toxicities were of Grades 1 and 2. Overall, these findings suggest that continuous sorafenib therapy and ondemand DEB-TACE allow excellent local disease control and do not lead to multiplicative toxicities.

Overall similar findings were reported in the final results of the Phase II, investigator-driven, prospective, single-arm multinational, UK Standardisation of Breast Radiotherapy trial. ${ }^{20}$ In total, 192 patients ( $81.5 \%$ of them in BCLC-B stage) received DEB-TACE with interrupted dosing of sorafenib (discontinued for 3 days before and 4-7 days after TACE) every 6-8 weeks. Combination of TACE and sorafenib was well tolerated, with only $8.1 \%$ of patients discontinuing due to adverse events. A mean of 2.7 TACE cycles were administered, and $52.6 \%$ of patients achieved complete response in target lesions. Median PFS was 12.8 months, and median TTP was 13.8 months, with an estimated 3 -year OS of $86.1 \%$. Last, a recently published meta-analysis of five studies (three randomized trials, one cohort study, and one prospective nonrandomized controlled trial, for a total of 899 patients) suggested the efficacy of the combination of TACE and sorafenib vs TACE alone. ${ }^{21}$ The HR for TTP was 0.75 (95\% CI: $0.48-1.03, p=0.003)$, and for OS, it was 0.76 (95\% CI: $0.47-1.05, p=0.147)$.

However, in a more restricted setting of patients, that is, those with main portal vein tumor thrombosis (MPVTT), outcomes were different. Zhang et al retrospectively evaluated 89 consecutive patients with BCLC-C HCC and MPVTT;22 45 of them received TACE and sorafenib, and the other 44 patients received sorafenib alone. The mean number of TACE sessions per patient was 2.6. The median durations of sorafenib in the combination group and sorafenib-only group were 5.6 and 5.4 months, respectively. DCR and TTP (3.0 vs 3.0) were similar between these two groups. Median OS was 7.0 months in the combination group and 6.0 months in the sorafenib-only group ( $p=0.54)$. No difference in the rate of sorafenib-related adverse events was reported. The authors concluded that, considering patients' morbidity after TACE, sorafenib monotherapy is appropriate for managing patients with advanced HCC and MPVTT.

Importantly, the Global Investigation of therapeutic DEcisions in hepatocellular carcinoma and Of its treatment with sorafeNib observational registry study of more than 3,000 HCC patients also provides information on TACE within combination schemes. ${ }^{23}$ In total, 3,202 patients were eligible for safety analysis, of whom 1,511 (47.2\%) patients 
underwent TACE prior to sorafenib and 325 (10.1\%) patients underwent TACE concomitantly. Adverse events were reported in $2,732(85.3 \%)$ patients, with no notable differences according to TACE treatment history. OS was 12.7 months in prior-TACE patients, 9.2 months in non-priorTACE subjects, 21.6 months in concomitant-TACE patients, and 9.7 months in non-concomitant-TACE patients.

Remarkably, in the major Single Pill to Avert Cardiovascular Events trial, patients with intermediate-stage multinodular HCC were randomly assigned to DEB-TACE (150 mg doxorubicin) plus sorafenib $(n=154)$ or placebo $(n=153) .{ }^{24}$ Median TTP with DEB-TACE + sorafenib was 5.6 months, compared with 5.5 months with DEB-TACE alone. ORRs for patients in the sorafenib and placebo groups were $55.9 \%$ and $41.3 \%$, respectively, and the DCRs were $89.2 \%$ and $76.1 \%$, respectively. No increase in adverse events was reported in the sorafenib group. The authors suggested that the combination of DEB-TACE and sorafenib is technically feasible, but it does not improve survival and tumor response when compared with DEB-TACE alone.

Sorafenib is not the only systemic agent tested for combination with TACE. In a small, randomized, Phase II study, Pinter et al investigated the efficacy and safety of cTACE and bevacizumab $(n=20)$ vs cTACE only $(n=20) .{ }^{25}$ Patients underwent cTACE with doxorubicin and intravenous administration of a placebo (cTACE-C) or bevacizumab (cTACE-B; $5 \mathrm{mg} / \mathrm{kg}$ ) every 2 weeks for 52 weeks. After the first TACE procedure, TACE was repeated twice in 4-week intervals if indicated and on demand thereafter. The trial was stopped prematurely for safety reasons. Overall, patients underwent a median of three TACE cycles and received 13 infusions of bevacizumab vs 11 infusions of the placebo. Severe septic $(n=8)$ and vascular $(n=9)$ side effects were observed in the combination group. Median OS was shorter in the combination group than that in the cTACE-only group (5.3 vs 13.7 months; HR =1.7; 95\% CI: 0.8-3.6) and became significant in patients with ChildPugh class A cirrhosis (7.3 vs 26.5 months; HR =2.6; 95\% CI: $1.0-6.6 ; p=0.049)$. There was no difference in radiologic response between the groups at 3, 6, or 12 months.

A pooled analysis of four retrospective studies assessed the survival outcome of TACE combined with RFA vs surgical resection in early HCC patients with resectable small tumor. ${ }^{26}$ Overall, TACE-RFA provided 1 - and 3-year OS and 1 -year recurrence-free survival rate compared with surgical resection, with a lower risk of major complications.

\section{Implications for clinical practice}

TACE, either conventional or DEB-based, remains the standard therapy for the treatment of HCC patients with BCLC-B disease. However, in recent years, its role has been challenged, due to lack of standardization and the high number of contraindications in this procedure. The use of alternative approaches, such as TARE, and of alternative staging systems (eg, the Hong Kong Liver Cancer staging system) has been suggested in this setting. ${ }^{27-29}$

In the past 2 years, clinical research has mainly considered TACE as a reference to test new TACE schemes or new therapeutic strategies or as a part of combination regimens.

HCC patients, and in particular patients at intermediatestage HCC, represent a heterogeneous population, characterized by varying tumor burden, liver function, and disease etiology. For this reason, a tailored approach is usually required to optimize the clinical outcomes for each subject. TACE represents the standard treatment approach in intermediate-stage HCC. However, a general consensus on the optimal schedule of TACE treatment is yet to be reached. In various studies, treatment was repeated at fixed intervals based on a preestablished number of courses or repeated on demand based on radiological response. DEB-TACE seems to reduce the frequency/severity of the post-TACE syndrome with respect to cTACE; however, no clear advantages in terms of survival were shown. Intriguing evidence appears to support the use of multiple chemotherapy agents within the single TACE procedure.

On the other hand, although indications are different and therefore a direct comparison does not appear immediately feasible, SIRT does appear to be at least as effective as TACE, with a similar safety profile. TACE was also inferior to curative treatments in a large, well-conducted database studies on patients with BCLC-B disease.

Despite these premises, due to the large clinical experience gained to date, TACE still has a role in clinical practice. Moreover, we believe that a deeper investigation into combination strategies should be recommended. In particular, the combination of TACE and sorafenib appears based on a very strong mechanistic rationale and has proved to be effective, especially in patients with BCLC-B disease, in most studies conducted to date. However, studies should further investigate the optimal timing of the embolization related to antiangiogenic therapy (concomitant vs sequential) and the ideal administration schedule in relation to different dosing regimens of sorafenib. Another intriguing line of research is the potential use of TACE and sorafenib in advanced-stage (BCLC-C) HCC patients. This approach led to promising results, but the optimal selection of suitable patients and the possibility of tailoring treatment schemes according to the specific needs should be better investigated in clinical 
studies. ${ }^{9}$ In particular, the combination of TACE and RFA merits consideration.

\section{Conclusion}

We believe that TACE will retain a role in clinical practice, especially within tailored combination regimens if the preliminary results obtained to date will be confirmed.

\section{Acknowledgment}

We thank Professor Michele Barone and Professor Roberto Eggenhoffner for their critical review of the manuscript.

\section{Disclosure}

The authors report no conflicts of interest in this work.

\section{References}

1. European Association for the Study of the Liver; European Organization for Research and Treatment of Cancer. EASL-EORTC clinical practice guidelines: management of hepatocellular carcinoma. $J$ Hepatol. 2012;56:908-943.

2. Cammà C, Schepis F, Orlando A, et al. Transarterial chemoembolization for unresectable hepatocellular carcinoma: meta-analysis of randomized controlled trials. Radiology. 2002;224:47-54.

3. Llovet JM, Bruix J. Systematic review of randomized trials for unresectable hepatocellular carcinoma: chemoembolization improves survival. Hepatology. 2003;37:429-442.

4. Raoul JL, Sangro B, Forner A, et al. Evolving strategies for the management of intermediate-stage hepatocellular carcinoma: available evidence and expert opinion on the use of transarterial chemoembolization. Cancer Treat Rev. 2011;37:212-220.

5. Sacco R, Antonucci M, Bargellini I, Marceglia S, Mismas V, Cabibbo G. Transarterial chemoembolization and sorafenib in patients with intermediate-stage hepatocellular carcinoma: time to enter routine clinical practice? Future Oncol. 2015;11:2371-2373.

6. Dufour JF, Bargellini I, De Maria N, De Simone P, Goulis I, Marinho RT. Intermediate hepatocellular carcinoma: current treatments and future perspectives. Ann Oncol. 2013;24(Suppl 2):ii24- ii29.

7. Cabibbo G, Maida M, Genco C, et al. Natural history of untreatable hepatocellular carcinoma: a retrospective cohort study. World J Hepatol. 2012;4:256-261.

8. Cabibbo G, Enea M, Attanasio M, Bruix J, Craxì A, Cammà C. A metaanalysis of survival rates of untreated patients in randomized clinical trials of hepatocellular carcinoma. Hepatology. 2010;51:1274-1283.

9. Cabibbo G, Tremosini S, Galati G, et al. Transarterial chemoembolization and sorafenib in hepatocellular carcinoma. Expert Rev Anticancer Ther. 2014;14:831-845.

10. Cabibbo G, Latteri F, Antonucci M, Craxì A. Multimodal approaches to the treatment of hepatocellular carcinoma. Nat Clin Pract Gastroenterol Hepatol. 2009;6:159-169.

11. Kloeckner R, Weinmann A, Prinz F, et al. Conventional transarterial chemoembolization versus drug-eluting bead transarterial chemoembolization for the treatment of hepatocellular carcinoma. BMC Cancer. 2015;15:465.

12. Liu B1, Huang JW, Li Y, et al. Single-agent versus combination doxorubicin-based transarterial chemoembolization in the treatment of hepatocellular carcinoma: a single-blind, randomized, phase II trial. Oncology. 2015;89:23-30.
13. Kolligs FT, Bilbao JI, Jakobs T, et al. Pilot randomized trial of selective internal radiation therapy vs. chemoembolization in unresectable hepatocellular carcinoma. Liver Int. 2015;35:1715-1721.

14. Pitton MB, Kloeckner R, Ruckes C, et al. Randomized comparison of selective internal radiotherapy (SIRT) versus drug-eluting bead transarterial chemoembolization (DEB-TACE) for the treatment of hepatocellular carcinoma. Cardiovasc Intervent Radiol. 2015;38: 352-360.

15. El Fouly A, Ertle J, El Dorry A, et al. In intermediate stage hepatocellular carcinoma: radioembolization with yttrium 90 or chemoembolization? Liver Int. 2015;35:627-635.

16. Pecorelli A, Lenzi B, Gramenzi A, et al. Curative therapies are superior to standard of care (transarterial chemoembolization) for intermediate stage hepatocellular carcinoma. Liver Int. 2016;37(3):423-433.

17. Sergio A, Cristofori C, Cardin R, et al. Transcatheter arterial chemoembolization (TACE) in hepatocellular carcinoma (HCC): the role of angiogenesis and invasiveness. Am J Gastroenterol. 2008;103: 914-921.

18. Dufour JF. TACE with or without systemic therapy? $J$ Hepatol. 2012;56:1224-1225.

19. Cosgrove DP, Reyes DK, Pawlik TM, Feng AL, Kamel IR, Geschwind JF. Open-label single-arm phase II trial of sorafenib therapy with drug-eluting bead transarterial chemoembolization in patients with unresectable hepatocellular carcinoma: clinical results. Radiology. 2015;277:594-603.

20. Chao Y, Chung YH, Han G, et al. The combination of transcatheter arterial chemoembolization and sorafenib is well tolerated and effective in Asian patients with hepatocellular carcinoma: final results of the START trial. Int J Cancer. 2015;136:1458-1467.

21. Hu MD, Jia LH, Liu HB, Zhang KH, Guo GH. Sorafenib in combination with transarterial chemoembolization for hepatocellular carcinoma: a meta-analysis. Eur Rev Med Pharmacol Sci. 2016;20:64-74.

22. Zhang Y, Fan W, Wang Y, et al. Sorafenib with and without transarterial chemoembolization for advanced hepatocellular carcinoma with main portal vein tumor thrombosis: a retrospective analysis. Oncologist. 2015;20:1417-1424

23. Geschwind JF, Kudo M, Marrero JA, et al. TACE treatment in patients with sorafenib-treated unresectable hepatocellular carcinoma in clinical practice: final analysis of GIDEON. Radiology. 2016;279: 630-640.

24. Lencioni R, Llovet JM, Han G, et al. Sorafenib or placebo plus TACE with doxorubicin-eluting beads for intermediate stage HCC: the SPACE trial. J Hepatol. 2016;64:1090-1998.

25. Pinter M, Ulbrich G, Sieghart W, et al. Hepatocellular carcinoma: a phase II randomized controlled double-blind trial of transarterial chemoembolization in combination with biweekly intravenous administration of bevacizumab or a placebo. Radiology. 2015;277:903-912.

26. Guo W, He X, Li Z, Li Y. Combination of transarterial chemoembolization (TACE) and radiofrequency ablation (RFA) vs. surgical resection (SR) on survival outcome of early hepatocellular carcinoma: a metaanalysis. Hepatogastroenterology. 2015;62:710-714.

27. Sacco R, Mismas V, Marceglia S, et al. Transarterial radioembolization for hepatocellular carcinoma: an update and perspectives. World $J$ Gastroenterol. 2015;21:6518-6525.

28. Sacco R, Conte C, Tumino E, et al. Transarterial radioembolization for hepatocellular carcinoma: a review. $J$ Hepatocell Carcinoma. 2016;3: 25-29.

29. Yau T, Tang VY, Yao TJ, Fan ST, Lo CM, Poon RT. Development of Hong Kong Liver Cancer staging system with treatment stratification for patients with hepatocellular carcinoma. Gastroenterology. 2014;146:1691-1700. 


\section{Publish your work in this journal}

The Journal of Hepatocellular Carcinoma is an international, peerreviewed, open access journal that offers a platform for the dissemination and study of clinical, translational and basic research findings in this rapidly developing field. Development in areas including, but not limited to, epidemiology, vaccination, hepatitis therapy, pathology and

molecular tumor classification and prognostication are all considered for publication. The manuscript management system is completely online and includes a very quick and fair peer-review system, which is all easy to use. Visit http://www.dovepress.com/testimonials.php to read real quotes from published authors.

Submit your manuscript here: https://www.dovepress.com/journal-of-hepatocellular-carcinoma-journal 